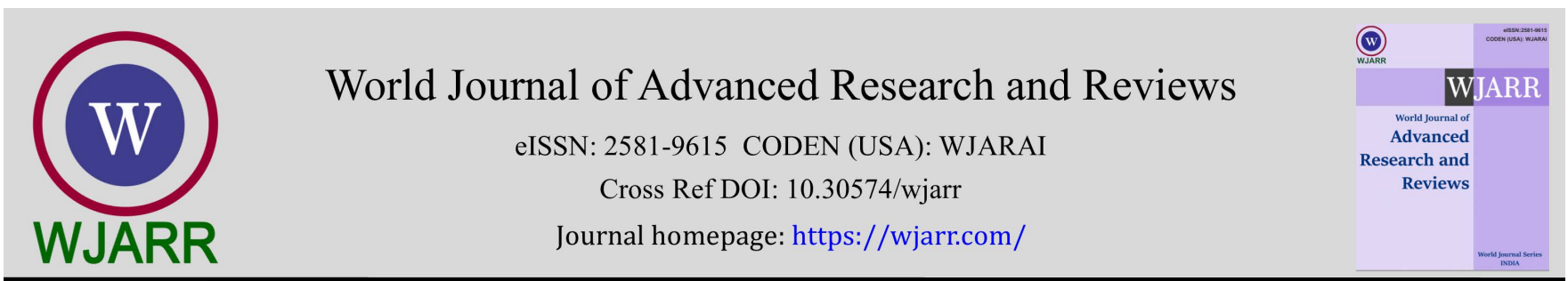

(RESEARCH ARTicle)

Check for updates

\title{
Assessment of intraoperative temperature management at the University Teaching Hospital of Kigali: A quality improvement project
}

\author{
Kaitlin M. Flannery ${ }^{1}$, Jean C. Uwimana ${ }^{2}$, Cesarie Nikuze ${ }^{3}$, Jean B. Uwineza ${ }^{4,}{ }^{*}$ and Ana M. Crawford 5 \\ ${ }^{1}$ Pediatric anesthesiologist Stanford University. \\ ${ }^{2}$ Anesthesiologist, University of Rwanda. \\ ${ }^{3}$ University Teaching Hospital of Kigali. \\ ${ }^{4}$ Anesthesiologist, intensivist. University of Rwanda. \\ ${ }^{5}$ Anesthesiologist, intensivist Stanford University.
}

World Journal of Advanced Research and Reviews, 2021, 09(01), 097-103

Publication history: Received on 21 December 2020; revised on 30 December 2020; accepted on 02 January 2021

Article DOI: https://doi.org/10.30574/wjarr.2021.9.1.0502

\begin{abstract}
Background: Due to the inhibition of thermoregulation by anesthetics, hypothermia occurs in $50 \%$ of patients undergoing general and neuraxial anesthesia without active warming. High-quality research has shown the negative effects of intraoperative hypothermia including increased rates of surgical site infections, increased hospital length of stay, increased cardiac morbidity and increased rate of transfusion. The University Teaching Hospital of Kigali commonly known as CHUK is the largest public referral hospital in Rwanda. The International Standards for Safe Anesthesia were not being met regarding intraoperative temperature management at CHUK. However, due to high ambient temperature in the operating room, it was unknown if this was a significant problem and worth investment, in a health system, with limited resources.
\end{abstract}

Methods: After institutional review board approval, a two-stage descriptive cross-sectional quality improvement project was performed. The first stage was to assess current management of intra-operative temperature, determine if there was a significant rate of hypothermia and establish risk factors for developing intra-operative hypothermia in this clinical setting. The second stage was to determine if locally available warming techniques, an underbody warming blanket, significantly reduced the incidence of intra-operative hypothermia.

Results: The primary result of the study was a $58 \%$ incidence of hypothermia with current standard practice. This was reduced to $6 \%$ with the use of an underbody water blanket. Temperature was measure intraoperatively in only $6 \%$ of cases and never documented. Adult patients and patients undergoing neuraxial anesthesia were more likely to experience hypothermia.

Conclusions: Intraoperative hypothermia is common at CHUK despite high ambient operating room temperature. Monitoring and documentation of temperature is not occurring on a regular basis. The use of underbody water body, though not the gold standard for intraoperative warming, was effective in this study and a realistic solution in an environment with limited resources.

Keywords: Intraoperative hypothermia; Underbody warming blanket; Incidence; Intraoperative warming

\footnotetext{
${ }^{*}$ Corresponding author: Jean ClaudeUwimana

M.D.Anesthesiologist,KIBOGORA district Hospital,uwijenclau9@gmail.com, phone number: +250782752037.
} 


\section{Introduction}

The International Standards for Safe Anesthesia were first developed by the World Federation of Societies of Anesthesiologists in 1992 and were updated, most recently, in 2010. The standards represent goals for the delivery of anesthesia globally. There are three levels outlined ranging from basic standards for rural hospitals to optimal standards for referral hospitals. Referral hospitals perform longer more complicated procedures requiring increased resources. The optimal standard for temperature management is the ability to measure temperature either continuously or at regular intervals. Optimal standards also require a strategy for maintaining normothermia [1].

Hypothermia is defined as a core temperature less than $36^{\circ} \mathrm{C}$. Without active warming during general and neuraxial anesthesia, half of patients' temperatures will nadir below $36^{\circ} \mathrm{C}$, with one third drifting below $35^{\circ} \mathrm{C}$ [2]. This is due to anesthesia induced inhibition of thermoregulation. The threshold for vasoconstriction and shivering is decreased by many anesthetics, including volatiles, nitrous oxide and propofol, allowing the body's normal temperature range to decrease by $2-4^{\circ} \mathrm{C}[3]$.

The negative effects of intraoperative hypothermia have been well documented. These include increased rate of surgical site infection, increased post-operative cardiac events, and coagulopathy with the need for increased transfusions. A study of 200 colorectal patients showed that hypothermic patients had infection rate of $18 \%$, with an average hospital stay 2.6 days longer, than normothermic patients with infection rate of 6\% [4]. The WHO includes intraoperative warming in their global guidelines for prevention of surgical site infections [5].Intra-operative normothermia was associated with a 55\% risk reduction of post-operative cardiac events, in patients at high cardiac risk [2]. A review looking at the effect of intraoperative hypothermia on transfusion requirement found a $22 \%$ increased risk of transfusion with hypothermia[6].

The University Teaching Hospital of Kigali (CHUK) is the largest public referral hospital in Rwanda. It is part of the University of Rwanda and the primary hospital for the training of anesthesiologists. The hospital has ten operating rooms. Standard monitors, including reusable oropharyngeal temperature probes are available in every operating room. The operating room temperature is not regulated. The average temperature in Kigali is $21^{\circ} \mathrm{C}$ and operating room temperature at $\mathrm{CHUK}$ is typically around $28^{\circ} \mathrm{C}$. Available warming techniques include warm IV fluids and blankets. Additionally there is one open infant incubator and one pediatric sized underbody water blanket.

The ASA Committee on Global Humanitarian Outreach and the Canadian Anesthesiologists' Society International Education Foundation have worked alongside the anesthesia faculty at the University of Rwanda since 2006, through the Overseas Teaching Program, to develop anesthesia residency didactics and improve access to safe physician-led anesthesia in Rwanda[7]. The University of Rwanda residency requires a quality improvement project similar to ACGME requirements. This partnership, has allowed for productive bilateral partnerships such as this quality improvement project.

\section{Material and methods}

This study included two descriptive cross-sectional stages. The first stage was to assess current management of intraoperative temperature, determine if there was a significant rate of hypothermia and establish risk factors for developing intra-operative hypothermia in this clinical setting. The second stage was to determine if locally available maximal warming techniques significantly reduced the incidence of intra-operative hypothermia. Approval of the study was obtained from the institutional review boards of the University of Rwanda and the University Teaching Hospital of Kigali. The study met institutional review board exception from Stanford University due to the quality improvement nature and low risk. Data was collected from February 2019 through April 2019.

In stage one of the study, oral informed consent was obtained from fifty patients or legal guardians, for patients under 18 years of age, undergoing elective or urgent surgery, with surgical length greater than thirty minutes, under general or neuraxial anesthesia. Patients presenting for emergency surgery, pregnant patients and patients who were febrile or hypothermic in the pre-operative area were excluded from the study. The patient's anesthetic and temperature management were at the discretion of the anesthesia team though resources were limited to reusable axial or oropharyngeal temperature probes for monitoring and warmed IV fluids and blankets for warming techniques. To assess temperature, a member of the study team obtained a forehead infrared skin temperature recording (EFT-161 LCD Digital Infrared Thermometer, AccuIT, New Delhi, India) within five minutes of the patient arriving to the PACU. To assess temperature monitoring, a member of the study team observed in the operating room and record if temperature monitoring was utilized. Temperature documentation was evaluated by reviewing the anesthesia record in the PACU. 
Additional information recorded on each patient included age, primary anesthesia type, surgical procedure and surgical length.

In stage two of the study, oral informed consent was obtained from the legal guardians of 50 pediatric patients undergoing elective or urgent surgery with surgical length greater than thirty minutes, under general or neuraxial anesthesia. Patients presenting for emergency surgery and patients who were febrile or hypothermic in the preoperative area were excluded from the study. The patient's anesthetic management was at the discretion of the anesthesia team. Intra-operative warming was performed with an underbody water blanket set at $38^{\circ} \mathrm{C}(\mathrm{Medwarm} 300$ D, Istanbul Medikal, Turkey) in addition to warmed IVF and blankets. A member of the study team measured forehead infrared skin temperature (EFT-161 LCD Digital Infrared Thermometer, AccuIT, New Delhi, India) every thirty minutes throughout the surgery and within five minutes of arriving in the PACU. Additional information recorded on each patient included age, primary anesthesia type, surgical procedure and surgical length.

\subsection{Statistical Analysis}

Primary analysis of stage one data is rate of postoperative hypothermia, temperature monitoring and temperature documentation. Sub-group analysis utilizing one-sided chi square tests and unpaired tests was performed to evaluate if there was statistically significant difference in rates of hypothermia based on age, primary anesthesia type, length of surgery or surgical subspecialty. Primary analysis of stage two data is rate of intra-operative hypothermia. Unpaired $t$ tests were used to compare incidence of hypothermia between patients in stage one and stage two.

\section{Results}

Table 1 Summary of Clinical Characteristics of Cross-Sectional Samples and Rates of Hypothermia

\begin{tabular}{|c|c|c|c|c|}
\hline & Stage One All & $\begin{array}{l}\text { Stage Two } \\
\text { All }\end{array}$ & $\begin{array}{l}\text { Stage One } \\
\text { Hypothermic }\end{array}$ & $\begin{array}{l}\text { Stage Two } \\
\text { Hypothermic }\end{array}$ \\
\hline \multicolumn{5}{|l|}{ Age (years) } \\
\hline $0-17$ & 19 & 50 & $8(42 \%)$ & $3(6 \%)$ \\
\hline$\geq 18$ & 31 & - & $21(67 \%)$ & - \\
\hline \multicolumn{5}{|l|}{ Anesthesia Type } \\
\hline General & 35 & 50 & $17(48.5 \%)$ & $3(6 \%)$ \\
\hline Neuraxial & 15 & - & $12(80 \%)$ & - \\
\hline \multicolumn{5}{|c|}{ Surgery Length (hours) } \\
\hline \multicolumn{5}{|l|}{$<2$} \\
\hline$<3$ & 12 & 39 & $5(42 \%)$ & $2(5 \%)$ \\
\hline $3-4$ & 18 & 5 & $12(67 \%)$ & $0(0 \%)$ \\
\hline \multirow[t]{2}{*}{$>4$} & 10 & 3 & $6(60 \%)$ & $1(33 \%)$ \\
\hline & 10 & 3 & $5(50 \%)$ & $0(0 \%)$ \\
\hline \multicolumn{5}{|l|}{ Surgical Specialty } \\
\hline General Surgery & 11 & 21 & $7(64 \%)$ & $1(5 \%)$ \\
\hline Neurosurgery & 3 & - & $2(67 \%)$ & - \\
\hline Orthopedic Surgery & 13 & - & 7 (54\%) & - \\
\hline Urology & 7 & 8 & $6(86 \%)$ & $0(0 \%)$ \\
\hline Otolaryngology & 6 & 19 & $4(67 \%)$ & $2(10 \%)$ \\
\hline Plastic Surgery & 4 & - & $1(25 \%)$ & - \\
\hline Ophthalmology & - & 2 & - & $0(0 \%)$ \\
\hline
\end{tabular}

Demographic and clinic characteristics of the subjects are listed in table above (Table1)

Given the resources available, mainly the pediatric sized underbody water blanket used for temperature management in stage two, the clinic characteristics of the two samples are quite different. All the subjects in stage two are pediatric 
patients and underwent general anesthesia. The surgery length in stage one was more varied while most surgeries in stage two were under two hours. Additionally, table 1 lists the characteristics of hypothermic subjects.

Among the 50 subjects in stage one who underwent anesthesia with no alteration in standard practice, $58 \%$ were hypothermic in the PACU. The temperature was monitored in only $6 \%$ of cases and was documented in the anesthesia record in $0 \%$ of cases. In none of the 20 surgeries over 3 hours in length was temperature ever measured.

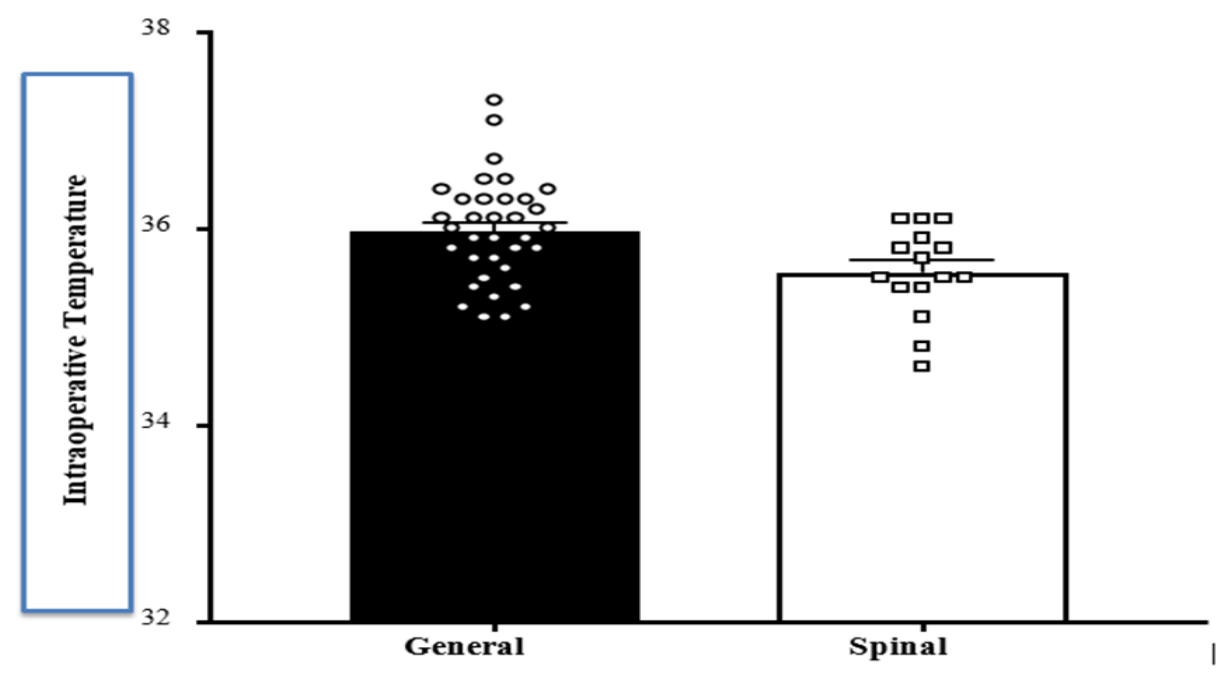

Figure 1 As seen in Figure 1, subjects who underwent neuraxial anesthesia were more likely to be hypothermic than subjects who received general anesthesia $(80 \%$ vs $48.5 \%$, $p$ value 0.01$)$. The mean temperature of patients undergoing neuraxial anesthesia was $35.5^{\circ} \mathrm{C}$ with a SEM of $0.1^{\circ} \mathrm{C}$ while the mean temperature of patients undergoing general anesthesia was $36^{\circ} \mathrm{C}$ with a SEM of $0.1^{\circ} \mathrm{C}$.

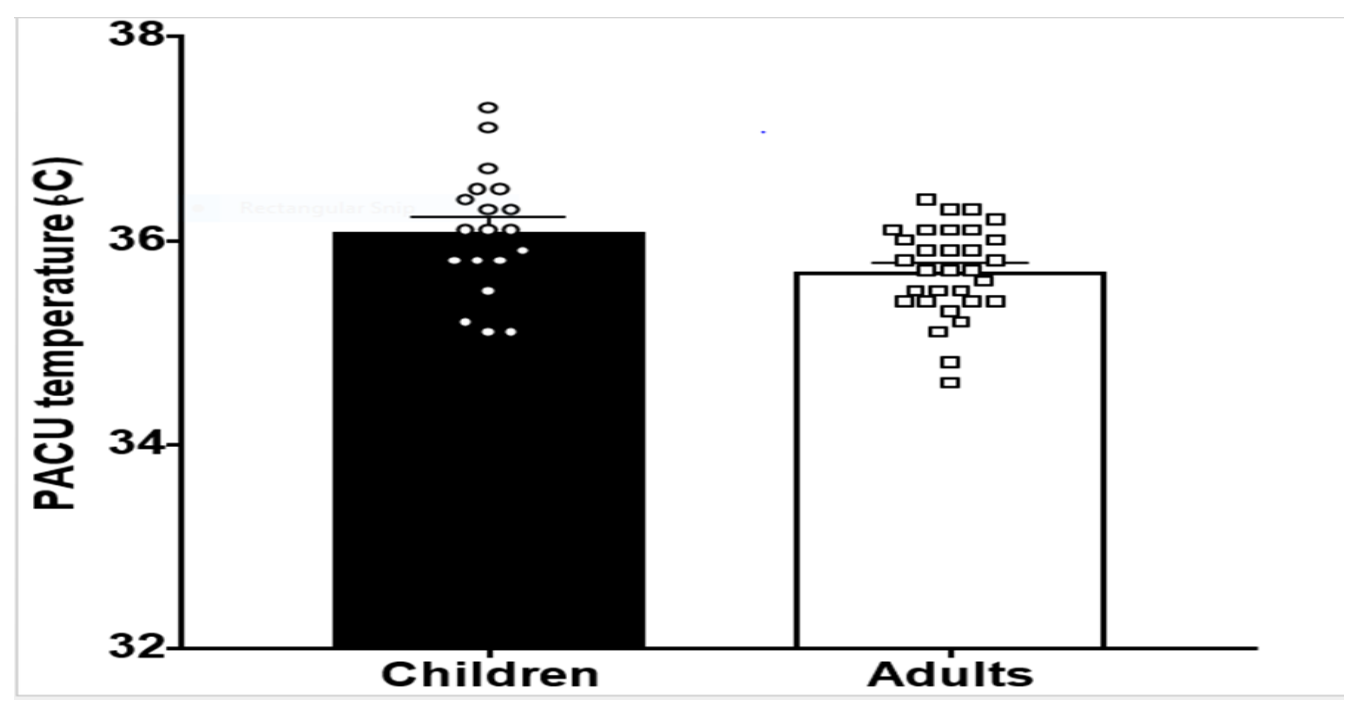

Figure 2 As seen in Figure 2, adult subjects were more likely to be hypothermic than children $(67 \%$ vs $42 \%$, p value 0.01). The mean temperature of adult patients was $35.7^{\circ} \mathrm{C}$ with a SEM of $0.1^{\circ} \mathrm{C}$ while the mean temperature of pediatric patients was $36^{\circ} \mathrm{C}$ with a SEM of $0.1^{\circ} \mathrm{C}$. No statistically significant differences were found in rates of hypothermia based on surgical length or subspecialty.

With the addition of an underbody water blanket to temperature management in stage two, the incidence of intraoperative and postoperative hypothermia was $8 \%$ and $6 \%$ respectively. The mean postoperative temperature in stage one was $35.8^{\circ} \mathrm{C}$ with a SEM of $0.5^{\circ} \mathrm{C}$ while the mean postoperative temperature in stage two was $36.6^{\circ} \mathrm{C}$ with a SEM of $0.3^{\circ} \mathrm{C}$. There is also a statistically significant difference in rate of hypothermia between pediatric subjects in stage one and stage two ( $42 \%$ vs $6 \%$, p value 0.01 ). Given the low rate of hypothermia in the stage two subjects, no subgroup analysis identified risk factors. 


\section{Discussion}

Development of this quality improvement project began after two cases in which significant hypothermia, $<35^{\circ} \mathrm{C}$ was identified, due to difficulty with pulse oximetry monitoring. Both cases were open abdominal surgeries for bowel resections with significant risk for surgical site infection. In these cases, temperature was not monitored until there was difficulty with pulse oximetry monitoring and there was not an active warm plan in place. International standards for safe anesthesia were not being met. The temperature in the operating rooms at CHUK is much warmer than the temperature in standard air-conditioned operating rooms in the global north. Due to this, it was presumed that intraoperative hypothermia was less common. The first stage of this project was to determine if there was a problem and to better define it. The results show that with current standard practice there is a significant rate of hypothermia. At least $58 \%$ of patients were hypothermic. In stage one, temperature was only measured in the PACU to avoid Hawthorne effect. The $42 \%$ of patients who were normothermic in the PACU could have experience intra-operative hypothermia. Stage one of this project showed that there are significant rates of perioperative hypothermia despite the increased ambient temperature. Risk factors identified include neuraxial anesthesia and adult patients though improvement is needed for all patients.

The second stage of this project was to determine if a reusable underbody water blanket significantly reduced the incidence of perioperative hypothermia. Prior research has shown water mattresses to be ineffective. The reason is thought to be a combination of minimal heat loss occurring between the patient and the mattress of a standard operating table and limited blood flow to the skin in contact with the blanket due to the weight on that dependent area [8].Water mattresses have also been associated with patient injury such as tissue burns [9]. This research is all several decades old. Research likely does not continue with these products because forced-air systems have been found to be more effective and have minimal risk for injury. The cost of forced-air warming is estimated at 10 USD per case [3]. This is cost-effective in the global north. However, it is cost prohibitive at CHUK. Additionally, lack of a reliable supply-chain makes use of disposable products challenging in low and middle-income countries. A realistic solution must be reusable and cost much less than 10 USD per case. This study showed a significant reduction in rate of postoperative hypothermia, $58 \%$ to $6 \%$, with the use of an underbody water mattress. Temperature of the water mattress was set to $38^{\circ} \mathrm{C}$ to reduce risk for any injury.

Given the significant body of research on the detrimental effects of intra-operative hypothermia, as anesthesiologists, we can improve the quality of care by maintaining intra-operative normothermia $[2,4,5,6]$. Looking specifically at CHUK, decreasing the rate of intra-operative hypothermia and the associated risks negative outcomes is important. Accurate reporting of surgical site infection rate is not available, however wound debridement is a common procedure at CHUK suggesting high rates of surgical site infection. Recent studies performed at CHUK have revealed high rates of antibiotic resistance $[10,11]$. Wound infections commonly grew S. aureus $(28 \%)$ and Klebsiella spp. (28\%). Resistance was common among the $\mathrm{S}$. aureus isolates with $82 \%$ resistant to oxacillin, $88 \%$ resistant to penicillin, $70 \%$ resistant to TMP/SMX and 6\% resistant to vancomycin[10]. Resistance was also common in the Klebsiella spp. with over 50\% resistant to third generation cephalosporins.[10] Many factors contribute to this growing resistance but significant among them is the limited antibiotic formulary available. Reducing the rate of infection and the need for antibiotics is important. Decreasing the risk of post-operative cardiac events is important, as the available treatment is limited to medical interventions. The nearest catheterization laboratory is not easy to access and there is currently one cardiothoracic surgeon in Rwanda. Blood transfusions are available, however supplies are limited.

There are several limitations to this study. Primary among them is the significant difference in the study population between the two stages. This was due to equipment limitations. However, statistically significant reduction in rate of hypothermia is also seen with comparison of only pediatric patients from stage one with stage two. An additional stage evaluating rates of hypothermia in adult CHUK patients with the utilization of underbody water blankets is necessary. Another limitation is the measurement of peripheral temperature during this study as opposed to core temperature. Infrared skin temperature is the measurement strategy used for temperature evaluation in the PACU globally and for this reason and its low risk and cost, it was used during this study. Outcomes such as surgical site infection rate or transfusion rate were not measured due to the robust data already available showing improvements in these areas with intraoperative normothermia.

The 2015 Lancet Commission on global surgery states that "surgery is an integral, indivisible component of a properly functioning healthcare system, and all people should have access to safe, high-quality surgical and anesthesia care with financial protection when needed"[12]. It is estimated that 5 billion people currently do not have appropriate access. The International Standards for Safe Anesthesia are available to guide anesthesiologists in proper management of patients [1]. A recent editorial in Anesthesiology, called for additional global anesthesia literature [13]. Many anesthesiologists from the global north participative in global health whether it be in capacity building, education or 
surgical mission work. The results of this study are significant as the importance of temperature monitoring and management in quality anesthesia may seem less important in areas where the ambient operating temperature is high and resources are low. However ignoring this standard ASA monitor results in suboptimal and inappropriate anesthesia care. A recent survey reports, 91\% of ACGME anesthesiology residents would like to participate in global health during residency [14].Currently, this most commonly takes the form of a service-based mission trip. This will often enhance the residents training experience but not provide a significant value to the community they briefly work in. This project highlights another option for residents interested in global anesthesia that can result in safer anesthesia for patients beyond the rotation.

\section{Conclusion}

In summary, with current practice, intraoperative hypothermia is common at CHUK despite high ambient operating room temperature. Application and documentation of standard temperature monitors is not occurring on a regular basis. Immediately actionable items include education of anesthesia care on the negative effects of intraoperative hypothermia and the importance of temperature monitoring and active warming. During the study, it was observed that patients under general anesthesia routinely had non-operative sites covered with warm blankets. This was not seen in patients undergoing neuraxial anesthesia. Therefore another immediate action is covering all patients with warm blankets. Next steps include submitting the results to the Ministry of Health to advocate for investment in warming equipment. Though force-air warmer would be ideal, underbody water blankets are realistic in this practice environment and this study showed a significant decrease in intraoperative hypothermia with its use. If investment in adult-sized underbody water blankets occur, it will be important to repeat this study and evaluate if rates of hypothermia decrease in the adult population.

\section{Compliance with ethical standards}

\section{Acknowledgments}

Vivianne L Tawfik, M.D., Ph.D., Anesthesiology, Perioperative and Pain Medicine Department, Stanford University, Stanford, CA, USA. Dr. Tawfik contributed through mentorship specifically in study design and statistics.

\section{Disclosure of conflict of interest}

The authors declare no competing interests.

\section{Statement of ethical approval}

Approval of the study was obtained from the institutional review boards of the University of Rwanda and the University Teaching Hospital of Kigali. The study met institutional review board exception from Stanford University due to the quality improvement nature and low risk.

\section{Statement of informed consent}

Informed consent was obtained from all individual participants included in the study.

\section{References}

[1] Merry AF, Cooper JB, Soyannwo 0, Wilson IH, Eichhorn JH: International standards for the safe practice of anesthesia 2010. Can J Anaesth. 2010; 57(11): 1027-34.

[2] Frank SM, Fleisher LA, Breslow MJ, Higgins MS, Olson KF, Kelly S, Beattie C: Perioperative maintenance of normothermia reduces incidence of morbid cardiac events: A randomized comparison trial. JAMA. 1997; 227(14): 1127-34.

[3] Sessler DI: Temperature Regulation and Monitoring, Miller's Anesthesia, 8th edition. Edited by Miller RD. Philadelphia, Saunders. 2015; 1622-46.

[4] Kurz A, Sessler DI, Lenhardt R. Perioperative normothermia to reduce the incidence of surgical-wound infection and shorten hospitalization: Study of wound infection and temperature group. NEJM. 1996; 334(19): $1209-15$.

[5] WHO: Global guidelines for the prevention of surgical site infections. Geneva, WHO. 2016. 
[6] Rajagopalan S, Mascha E, Na J, Sessler DI: The effects of mild perioperative hypothermia on blood loss and transfusion requirements. Anesthesiology. 2008; 108(1): 71-7.

[7] Banguti PR, Uwambazimana JD, Crawford AM: ASA Global Humanitarian Outreach Overseas Teaching Program: Rwandan vs. American Perspective. ASA Monitor. 2017; 81: 46-50.

[8] Morris RH, Kumar A: The effect of warming blankets on maintenance of body temperature of the anesthetized paralyzed adult patient. Anesthesiology. 1972; 36(4): 408-11.

[9] Crino MH, Nagel EL. Thermal burns caused by warming blankets in the operating room. Anesthesiology. 1968; 29(1): 149-50.

[10] Ntirenganya C, Manzi O, Muvunyi CM, Ogbuagu O: High prevalence of antimicrobial resistance among common bacterial isolates in a tertiary healthcare facility in Rwanda. Am J Trop Med Hyg. 2015; 92(4): 865-70.

[11] Rickard J, Ngarambe C, Ndayizeye L, Smart B, Riviello R, Majyambere JP, Ghebre RG: Antibiotic use and antimicrobial resistance of surgical patients with peritonitis at a tertiary referral hospital in Rwanda. Surg Infect (Larchmt). 2018; 19(4): 382-7.

[12] Meara JG, Hagander L, Leather AJM: Surgery and global health: a Lancet Commission. Lancet. 2014; 383(9911): 12-13.

[13] Harris MJ: We need more reports of global health anesthesia articles. Anesthesiology. 2016; 124: 167-9.

[14] McCunn M, Speck RM, Chung I, Atkins JH, Raiten JM, Fleisher LA: Global health outreach during anesthesiology residency in the United States: a survey of interest, barriers to participation and proposed solutions. J Clin Anesth. 2012; 24(1): 38-43. 